\title{
FUSION OF THERMAL AND RGB IMAGES FOR BORDER SECURITY SURVEILLANCE
}

\author{
ANKUSH RAI*, JAGADEESH KANNAN R \\ School of Computing Science and Engineering, VIT University, Chennai, Tamil Nadu, India. Email: ankushressci@gmail.com
}

Received: 13 December 2016, Revised and Accepted: 03 April 2017

\begin{abstract}
Objective: Border security surveillance in remote areas are often suffer from low visibility and poor lighting conditions thus, tracking and recognizing the intruder in such a situation is a daunting task. In this study, a fusion technique is presented for enhancing the visibility by merging feature sets from thermal and RGB image sequence.

Method: we have proposed a methodology for video processing that combines the advantages of motion detection, predictability, and objects segmentation simultaneously for adaptive land cover change detection and prediction. The technique also solves the problem of computing the feature sets in massive sequences of images by carry forwarding the past feature sets for the consequent image sequence.

Results and Discussion: This methodology of automated scene surveillance presented in the paper is devoid of constraints such as propagating imperfection. Once, the similar features are transmitted to the prediction model that relies on the changes on the associated cluster points and consequently update the model. The results derived presented in this study shall help boost more reliable decisions by the automated intelligence in military and rescue operations.
\end{abstract}

Keywords: Multi-Image fusion, Security surveillance, Motion detection, Position estimation, Prediction.

(C) 2017 The Authors. Published by Innovare Academic Sciences Pvt Ltd. This is an open access article under the CC BY license (http://creativecommons. org/licenses/by/4. 0/) DOI: http://dx.doi.org/10.22159/ajpcr.2017.v10s1.19685

\section{INTRODUCTION}

In the past, few studies have been concentrated on predicting land cover changes while moderately considering the imperfect aspect of data $[1,2]$. Based on which the imperfection is treated on three levels: The input data, the model and model outputs. However, the propagation of imperfections from one level to another is not well addressed. The change prediction for such tasks is often marked by several types of imperfections which gradually influence the accuracy required to make decisions. However, the source of this imperfection cannot always be resultant from an existing inconsistency, but it may also be contributed to it due to ambiguity and lack of precision [3-5]. Decisions on the land cover could change consequently, and yet those approach was based on sensitivity analysis and imperfection propagation methods but lacks efficient data processing to bring out precise results; such that they did not qualifies for the real-time application in surveillance environments. In this study, we present a novel approach to accomplish the automation and efficient image processing for data deduction from scene surveillances.

\section{METHODS}

The process of scene surveillance is generally subjected to manual visual references, but in the context of automation it suffers several types of imperfections which affect the reliability of decision about these changes [6,7]. Although we proposed a new model for automated scene surveillance where a drastic improvement is done over video processing. In earlier models, the surveillance is restricted to object segmentation or requires a hefty chunk of programing for the training of programing modules and yet have bear imperfection related to the input of models and its propagation through models $[8,9]$. To simplify this gap between manual surveillance and automated scene surveillance, we have proposed a methodology for video processing that combines the advantages of motion detection, predictability, and objects segmentation simultaneously for adaptive land cover change detection and prediction. The proposed methodology entitled as frame propagation from massive sequencization fusion (FPMSF) which consists of following steps as shown in the flowchart of the proposed algorithm in Fig. 1.

\section{Algorithm: FPMSF}

Input: Input video frame $\{\mathrm{x} 1 \ldots \mathrm{xn}\}$

Output: Tagged local pattern sets (LPS)

Step 1: Perform data mapping of the total number of colored units extended in two-dimensional axis and cluster mapping pattern of the colored sets.

Step 2: Mappe Multiscale object regions of objects within the frame using Attribute based level adaptive thresholding algorithm [6].

Step 3: Evaluate.

$\mathrm{P}(\overrightarrow{\mathrm{X}})=\prod_{\mathrm{i}=1}^{\mathrm{n}} \mathrm{f}\left(\mathrm{x}_{\mathrm{i}}, \varphi\right)$

Correlation of cluster points $=\sum_{x, y} \frac{\left(x-\mu_{x}\right)\left(y-\mu_{x}\right) P(x, y)}{\gamma_{x} \gamma_{y}}$

Homogenity of cluster points of annotated frame $\mathrm{P}^{\prime}=\sum_{\mathrm{x}, \mathrm{y}} \frac{\mathrm{P}(\mathrm{x}, \mathrm{y})}{1+|\mathrm{x}-\mathrm{y}|}$

Where, $\mathrm{x}$ and $\mathrm{y}$ are the two consequent video frames, $\mathrm{P}$ and $\mathrm{P}^{\prime}$ are the non-annotated and frame, $\mu_{x}$ and $\mu_{y}$ are the histogram profiling of the subsequent frames $x$ and $y$ in association. Furthermore, $\varphi$ is the rate of the change of cluster points from subsequent frames given by,

$$
\gamma_{x}=\frac{1}{X} \sum_{i=1}^{n} x_{i}-x_{i-1}
$$

Step 4: Thus, the most prominent value of the correlation between cluster points validates the given frame into annotated frame or tagged from the database or by the user. Thus, the non-annotated frame $\mathrm{P}$ is discarded, and thus we have fusion sequence frames $\vec{X}=\left[\begin{array}{c}\mathrm{x}_{1} \\ : \\ \mathrm{x}_{\mathrm{n}}\end{array}\right]$ which 


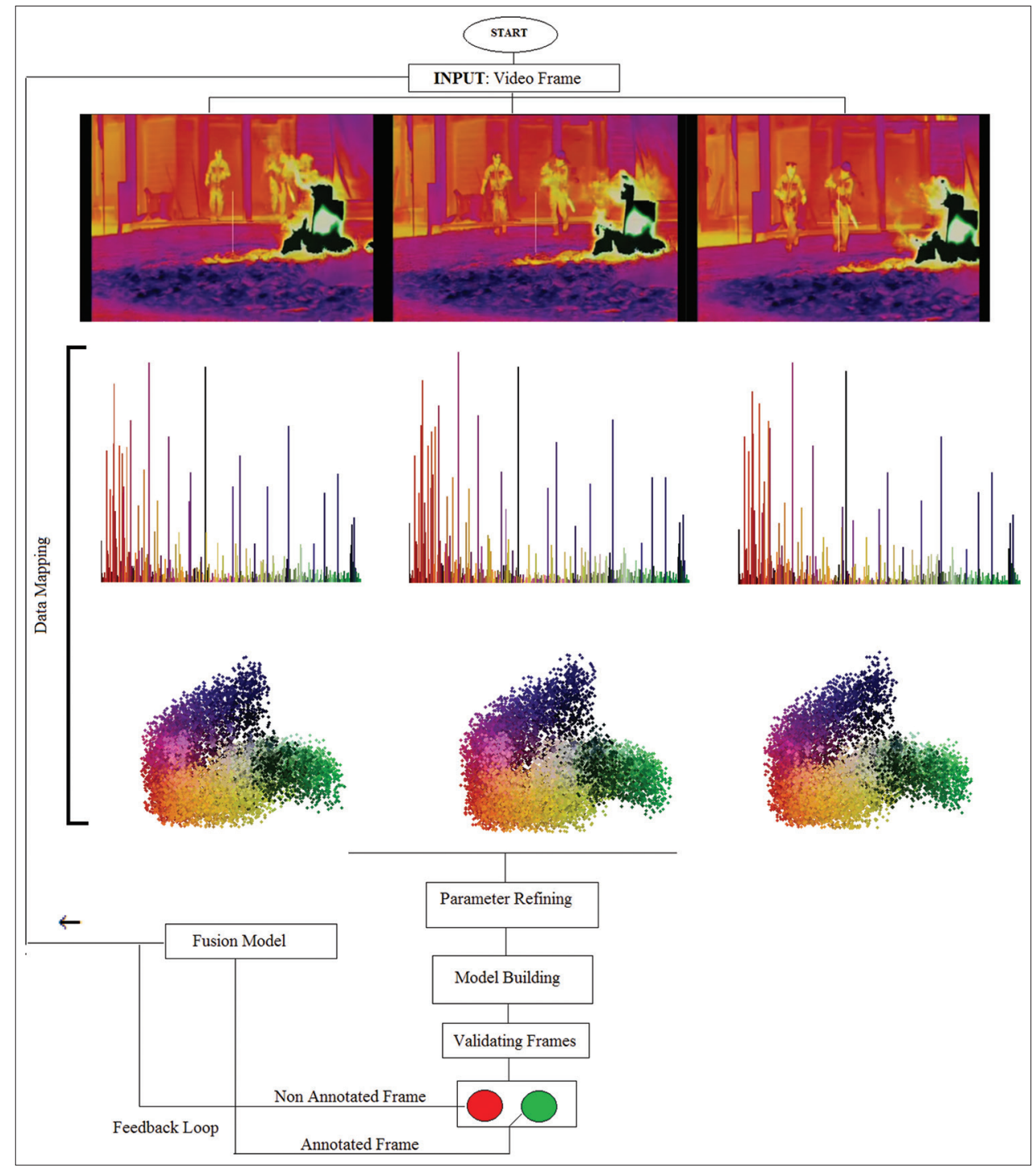

Fig. 1: Flowchart of the proposed algorithm

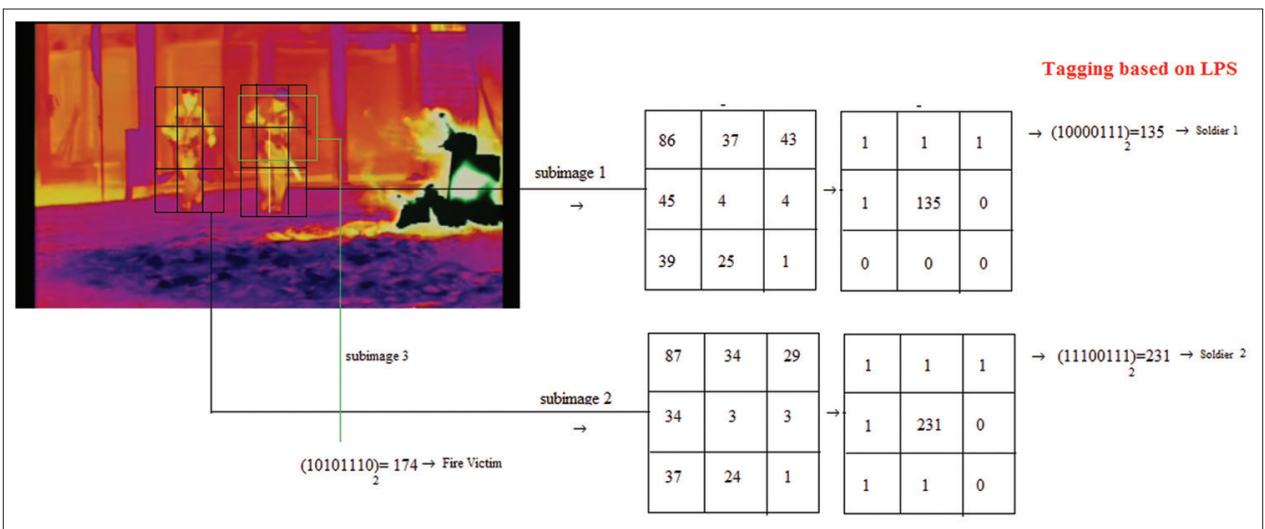

Fig. 2: Automated tagging of moving objects from by the proposed algorithm

are the operational vector of the paired annotated frames.

Step 5: Idealized LPS (model development) of the above-sequencized frame of the operational vector of the paired annotated frames is given by,

$$
\operatorname{LPS}(\mathrm{x}, \mathrm{y})=\sum_{\mathrm{P}=0}^{\mathrm{P}^{\prime}-1} \mathrm{~S}\left(\left(\gamma_{\mathrm{x}}-\gamma_{\mathrm{y}}\right)^{2^{\mathrm{P}^{\prime}}}\right)
$$

$$
\text { Where } s(x)=\left\{\begin{array}{c}
1, \text { if } \varphi \text { is maximal } \\
0, \text { otherwise }
\end{array}\right.
$$

Which sets the tagging of the object as shown in Fig. 2 and $\mathrm{s}(\mathrm{x})$ validates the pattern.

Step 6: Send feedback loops to $\overrightarrow{\mathrm{X}}$. 


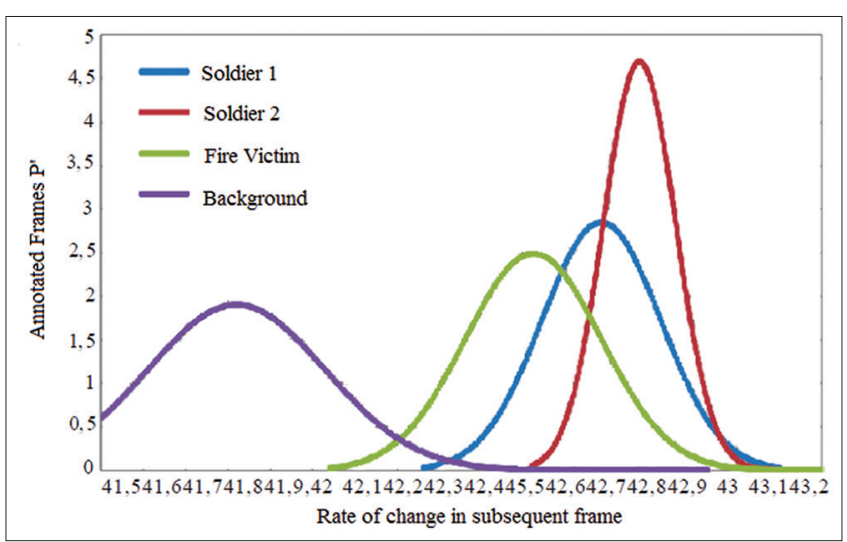

Fig. 3: Comparison of rate of change of subsequent frames with respect to the annotated frame from the fusing model of sequencized images

\section{CONCLUSION}

This methodology of automated scene surveillance presented in the paper is devoid of constraints such as propagating imperfection. Throughout the fusion model for scene change prediction methodology relies on computing membership functions for input features for a given landscape (Fig. 3). Then, these fusing membership functions are evaluated through a sensitivity analysis module to tag, alert and identify the most influential features in the overall scenario. After that similar features are transmitted to the prediction model that relies on the changes on the associated cluster points and consequently update the model. This helps to identify the optimal values of those sets of features that reduce the overall imperfection. Finally, the algorithm explicitly trained itself over the time to take and mold all attributes describing a particular object while considering the optimal values of influential attributes. The implication of this study suggests that the work achieved in this study shall help boost more reliable decisions by the automated intelligence in military and rescue operations.

\section{REFERENCES}

1. Aksoy $\mathrm{S}$, Cinbis RG. Image mining using directional spatial constraints. IEEE Geosci Remote Sens Lett 2010;7(1):33-7.

2. Hubert-Moy L, Corgne S, Mercier G, Solaiman B. Land use and land cover change prediction with the theory of evidence: A case study in an intensive agricultural region of France. Fifth International Conference on Information Fusion; 2012. p. 114-21.

3. Boulila W, Farah IR, Ettabaa KS, Solaiman B, Ben Ghézala H. A data mining based approach to predict spatio-temporal changes in satellite images. Int J Appl Earth 2011;13(3):386-95.

4. Boulila W, Farah IR, Ettabaa KS, Solaiman B. Combining decision fusion and uncertainty propagation to improve land cover change prediction in satellite image databases. J Multimed Proc Technol 2011;2(3):127-39.

5. Boulila W, Farah IR, Ettabaa KS, Solaiman B, Ben Ghézala H. Spatiotemporal modeling for knowledge discovery in satellite image databases. CORIA 2010: Conference en Recherche d'Information et Applications, Sousse, 2010. p. 35-49.

6. Rai A. Attribute based level adaptive thresholding algorithm for object extraction. J Adv Robot 2015;12:64-8.

7. Rai A. Shell implementation of neural net over the UNIX environment for file management: A step towards automated operating system. J Oper Syst Dev Trends 2014;12:10-4.

8. Rai A. Air computing: A parallel computing module for offloading computational workload on neighboring android devices. Recent Trends Parallel Comput 2015;13:10-3.

9. Rai A, Ramanathan S. Distributed learning in networked controlled cyber physical system. Int J Pharm Technol 2016;8(3):18537-46. 